\title{
Modifikation der radioimmunologischen Insulinbestimmung nach Hales und Randle
}

\author{
H.-J. QUABBE
} II. Med. Univorsitëtsklinik (Direktor: Prof. Dr. M. SctwaB) und Nuklcarmedizinische Abteilung (Leiter: Prof. Dr.
K. OfwF) der Freien Universität Berlin

Fingegangen am 22. Juli 1968

Modification of the Procedure of Hales and Randle for the Radioimmunoassay of Insulin.

Summary. A modification of the radioimmunoassay for insulin (method of HALES and RANDLE) is described. Separation of precipitated/antibody-bound insulin from free insulin was achieved by contrifugation instead of filtration. Addition of a carrier-immunoprecipitate relatively diminished possiblo losses by doeantation during the washing steps. Sensitivity $(10 \mu \mathrm{C} / \mathrm{ml})$, preeision $(\lambda=$ $0.067-0.314$ ) and reproducibility are comparable with some other published data. The modification avoids the difficulties due to the filtration procedure in the use of commercially available test-kits for the radioimmunoassay of insulin.

Modification du dosage radioimmunologique de l'insuline selon Hales et Randle.

Résumé. La méthode de Hanes et Rander du dosage radioimmunologique de l'insuline a été modifiée. La séparation du complexe précipité (insuline anticorps) de l'insuline libre a été effectuée par centrifugation au lieu de la filtration. L'addition d'un immunoprécipité transporteur diminue l'importance des pertes pendant le lavage du précipité. La sensibilité $(10 \mu \mathrm{U} / \mathrm{ml})$, la précision $(\lambda=$ $0.067-0.314)$ et la reproductibilité permettent une com- paraison favorable avoo des résultats publiés dans la littérature. La modification permet d'utiliser des combinaisons commercialisées pour le dosage radioimmunolo. gique de l'insuline tout en évitant les difficultés que peut poser la séparation $« \mathrm{~B} / \mathrm{F} »$ par la filtration.

Zusammenfassung. Fs wird eine Modifikation der radioimmunologischen Insulinbestimmung nach HALES und RaNdLE angegeben. Die Trennung von präzipitiertem/antikörpergebundenem und freiem Insulin wird nicht durch Filtration, sondern durch Zentrifugation vorgenommon. Durch Zusatz eines Träger-Immunopräzipitates wird eine Präzipitatmenge erzielt, die groß genug ist, um den Einfluß eventueller Verluste während des Waschens und Dekantierens genügend klein zu halten. Sensibilität $(10 \mu \mathrm{E} / \mathrm{ml})$, Genauigkeit $(\lambda=0.067-0.314)$ und Reproduzierbarkeit erlauben einen zufriedenstellenden Vergleich mit entsprechenden Angaben der Jiteratur. Die Modifikation ermöglicht die Benutzung käuflich erhältlicher Testkombinationen zur radioimmunologischen Insulinbestimmung unter Vermeidung der Sehwierigkeiten, die häufig bei der Benutzung der Filtration für die B/F. Trennung auftreten.

Key-words. Radioimmunoassay, insulin, B/F-separation, index of precision
Eine radioimmunologische Methode zur Bestimmung des Insulins wurde zuerst von YALOW und BERsoN angegeben [11]. Ihr Prinzip ist folgendes: Eine konstante Menge Insulin-Antiserum wird inkubiert mit den zu bestimmenden Plasmaproben und einer kleinen Menge radioaktiv-markierten Insulins, die in allen Ansatzröhrchen identisch ist. Endogenes Insulin des Plasmas und markiertes Insulin konkurrieren um die in begrenzter Anzahl vorhandenen Bindungsstellen des Antikörpers. Je mehr endogenes Insulin vorhanden ist, desto weniger markiertes Hormon wird an den Antikörper gebunden. Für eine Standardkurve werden die Plasmaproben durch eine Reihe von Insulin-Lösungen verschiedener, bekannter Konzentrationen ersetzt. Am Endo der Inkubation werden Antikörper-gebundenes und freies Insulin voneinander getrennt (z.B. durch Elektrophorese, durch Differentialadsorption oder durch Immunpräzipitation der Gamma-Globuline). Die Radioaktivität des Antikörper-gebundenen Insulins wird gemessen und als Prozent der ursprüng. lich zugegebenen Gesamtradioaktivität berechnet (alternativ kann das Verhältnis des gebundenen Insulins zum freigebliebenen (B/F) berechnet werden). In einem Koordinatensystem wird die gebundene Radioaktivität

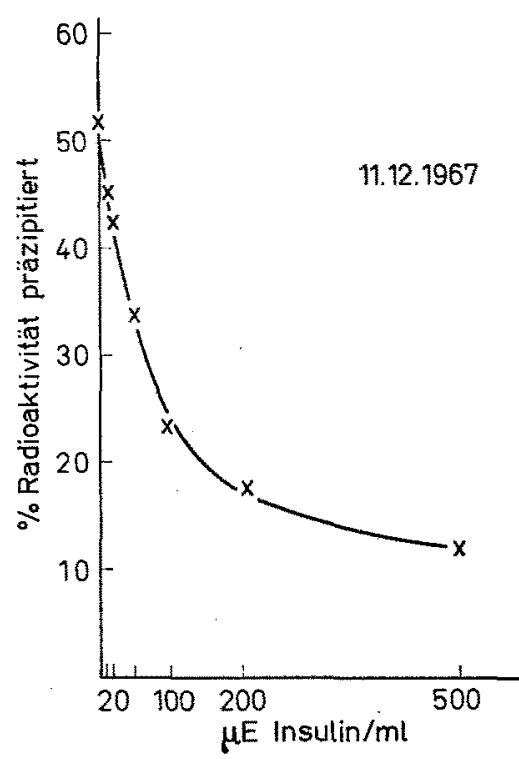

Abb. 1. Insulin Standardkurve, Diese Standardkurve wurde unter Benutzung der im Text beschriebenen modi. fizierten Methode gewonnen. Für die Herstellung der Insulin-Standardlösung wurde Schweine-Insulin verwendet 
(Ordinate) $\mathrm{zu}$ der jeweils zugehörigen Menge unmarkierten Insulins der Standardlösung (Abszisse) in Beziehung gesetzt (Konstruktion der Standardkurve, s. Abb. 1). Von dieser Standardkurve können die Insulinkonzentrationen der Plasmaproben abgelesen werden.

In vielen Laboratorien wird heute die käuflich erhältliche Testkombination des Radiochemical Centre, Amersham (England) benutzt, deren Arbeitsanweisung ${ }^{1}$ im wesentlichen der Variation C einer von HaLES und RANDLE [5] beschriebenen Methode entspricht. Sie weist folgende Besonderheiten auf:

$$
\begin{gathered}
\text { Prä-präzipitiertes Anti-Insulin-Serum (0.1 ml) } \\
\text { (Meerschweinchen/Kaninchen) } \\
+ \\
\text { Plasma oder Standard-Insulin-Lösung }(0.1 \mathrm{ml}) \\
(0-10-20-50-100-200-500 \mu \mathrm{E} / \mathrm{ml}) \\
\mid \begin{array}{l}
\text { Inkubation } \\
\left(24 \mathrm{Std} /+4^{\circ} \mathrm{C}\right)
\end{array}
\end{gathered}
$$

2. Der Insulin-Antikörper (der vom Meerschweinchen stammt) wird mittels eines Anti-Gammaglobulinserums (vom Kaninchen) präzipitiert. Diese Präzipitation erfolgt schon vor der Inkubation mit den Insulinstandardlösungen bzw. den Plasmaproben ${ }^{2}$. Dadurch soll der Einfluß einer unspezifischen Kreuzungsrealktion zwischen dem präzipitierenden Anti-Meerschweinchen-Gammaglobulin-Serum und Gammaglobulin des menschlichen Plasmas verhindert werden. Der so ,präpräzipitierte" Insulin-Antikörper verliert nicht seine Fähigkeit, das Antigen (Insulin) zu binden.

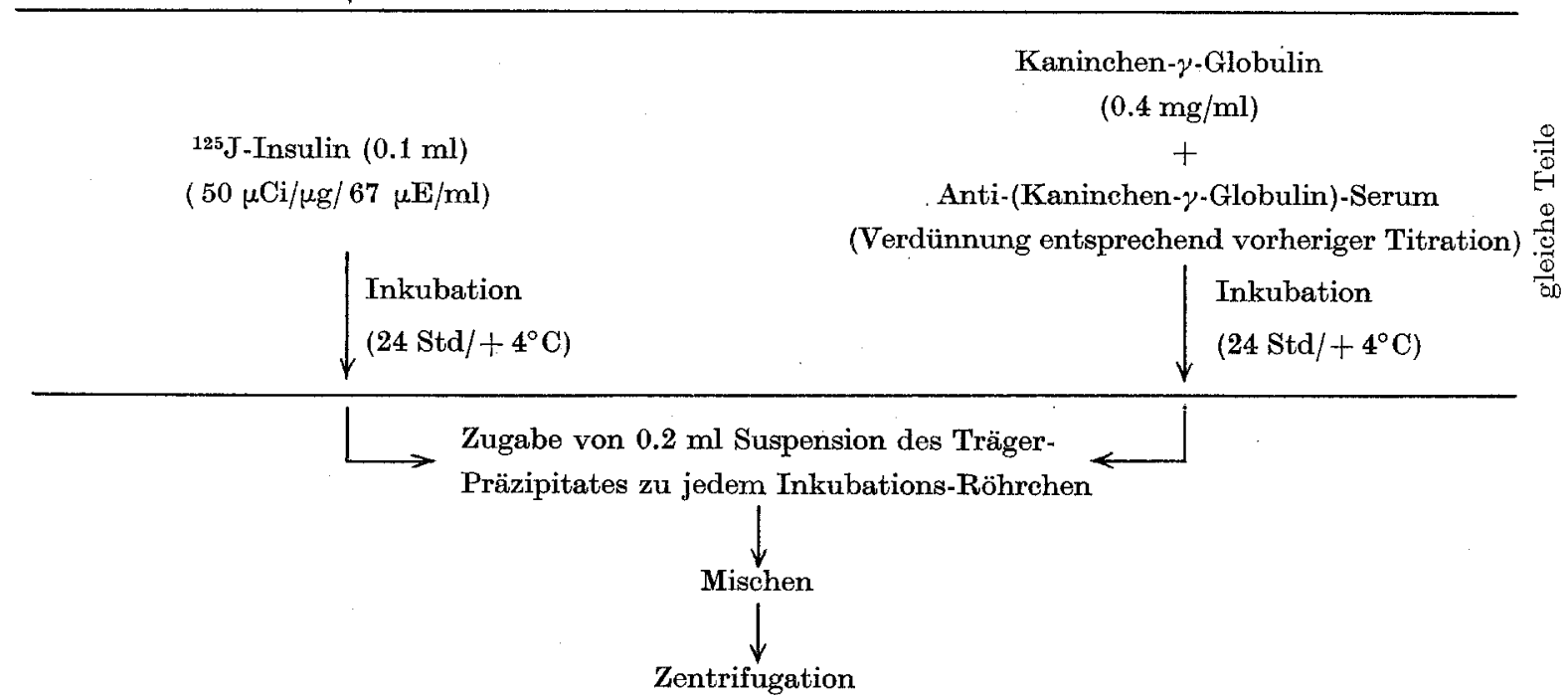

(30 Min bei $/ 3000 \mathrm{~g}$ )

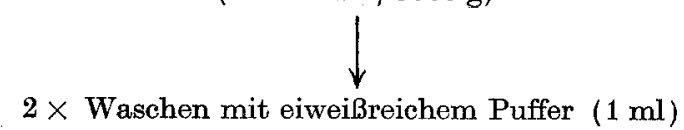

(5 mg Bovines Serum-Albumin pro ml) (jeweils zentrifugieren)

Abb. 2. Schema der modifizierten Insulinbestimmungsmethode unter Benutzung der Zentrifugation zur B/F-Trennung

1. Die Inkubation des Insulin-Antikörpers findet zunächst nur mit dem unmarkierten Insulin der Standardlösung bzw. mit den Plasmaproben statt. Das radioaktiv markierte Insulin wird erst zum Ansatz hinzugefügt, wenn die Bindung des unmarkierten Insulins an den Antikörper weitgehend stattgefunden hat. Die Sensibilität der Methode wird hierdurch erhöht.

1 Data sheet 5616, The Radiochemical Centre, Amersham.

2 In der Testkombination des Radiochemical Centre ist das bereits präzipitierte Insulin-Antiserum als ,Binding Reagent" enthalten.
3. Die Trennung des an den Antikörperkomplex gebundenen vom freien Insulin (B/F-Trennung) am Ende der Inkubation geschieht durch Filtration auf „Millipore"- oder „Oxoid"-Filtern. Hierbei passiert das freie Insulin das Filter, und der präzipitierte Insulin-Antikörperkomplex wird zurückgehalten.

4. Nach den Angaben des Radiochemical Centre reagiert der benutzte Insulin-Antikörper in gleicher Weise mit Human-, Rinder- und Schweine-Insulin.

Die Inkubation für die Insulinbestimmungen, wie sie vom Radiochemical Centre empfohlen wird, ist danach folgendermaßen: Ein mit Antigammaglobulin- 
Serum präzipitiertes Anti-Insulinserum (,,Binding Reagent") wird mit den Plasmaproben oder Standardinsulinlösungen verschiedener Konzentrationen bei $+4^{\circ} \mathrm{C}$ inkubiert. Nach 6 Std wird das radioaktiv markierte Insulin hinzugefügt, und eine zweite Inkubation von $18 \mathrm{Std}$ bei $+4^{\circ} \mathrm{C}$ angeschlossen. Danach wird die B/F-Trennung durch Filtration vorgenommen. Die Radioaktivität der Filter wird gemessen (sie entspricht der Fraktion des antikörpergebundenen Insulins). Die Konstruktion der Standardkurve und die Ablesung der unbekannten Insulinkonzentrationen erfolgt wie üblich.

Die Methode der Abtrennung des präzipitierten Insulin-Antikörperkomplexes durch Filtration ist relativ zeitraubend. Außerdem ergab sie in eigenen Versuchen häufig sehr schlechte Doppel- bzw. DreifachWerte. Hierfür könnten verschiedene Porengrößen der Filter, Adsorption ungebundenen Insulins an die Filter und andere Ursachen angeschuldigt werden, jedoch wurden nähere Untersuchungen nicht vorgenommen. Schwierigkeiten bei Benutzung der Filtration zur B/F'Trennung bei radioimmunologischen Hormonbestimmungen wurden auch von anderer Seite berichtet $[6$, $10^{3}$ ]. Es soll im folgenden eine Modifikation beschrieben werden, bei der unter Benutzung der Testkombination des Radiochemical Centre, Amersham, die Filtration durch eine Zentrifugation ersetzt wurde. Zugleich wurden die Inkubationszeiten der Originalmethode ( 6 und $18 \mathrm{Std}$ ) so verändert, daß alle Arbeitsgänge morgens um 8 Uhr begonnen und damit auch bei größeren Ansätzen innerhalb der normalen Laborarbeitszeit beendet werden können (zweimal $24 \mathrm{Std}$ ).

\section{Methodik}

\section{Ansatzschema (s. Abb. 2)}

Der Ansatz wird zunächst nach der Arbeitsvorschrift des Radiochemical Centre durchgeführt; es wird lediglich die Zeit der Inkubation verlängert: $0.1 \mathrm{ml}$ vor-präzipitierter Insulin-Antikörper (Binding Reagent) werden mit $0.1 \mathrm{ml}$ Insulin-Standardlösung bzw. $0.1 \mathrm{ml}$ Plasma $24 \mathrm{Std}$ lang bei $+4^{\circ} \mathrm{C}$ inkubiert. Dann werden $0.1 \mathrm{ml}$ radioaktiv markierten Insulins zugegeben, und es wird für weitere 24 Std bei $+4^{\circ} \mathrm{C}$ inkubiert. Zu Beginn dieser zweiten Inkubationsperiode wird jedoch getrennt hiervon ein zusätzliches präzipitierendes Antigen-Antikörper-System bei $+4^{\circ} \mathrm{C}$ inkubiert (,Trägerpräzipitat"c ${ }^{c}$ s. unten). Die Präzipitation dieses Systems ist nach 24 Std abgeschlossen. Nach Beendigung der zweiten Inkubationsperiode von 24 Std werden $0.2 \mathrm{ml}$ Suspension dieses Trägerpräzipitates zu jedem Röhrchen des Ansatzes (Standardkurve, Plasmaproben, Kontrollen) hinzugefügt. Unmittelbar

3 Seit kurzem werden vom Radiochemical Centre, Amersham, neue Oxoid-Filter empfohlen, die nach speziellen Angaben hergestellt und vor der Abgabe auf ihre Brauchbarkeit für die Bestimmungsmethode geprüft werden. anschließend wird zentrifugiert (30 Min. bei etwa $3000 \mathrm{~g},+4^{\circ} \mathrm{C}$. Kürzere Zentrifugationszeiten haben sich nicht bewährt). Das an den Antikörperkomplex gebundene Insulin sedimentiert zusammen mit dem Trägerpräzipitat, während das freie Insulin im Überstand bleibt. Nach Dekantieren wird das Präzipitat zweimal mit jeweils $1 \mathrm{ml}$ eines eiweißreichen Puffers (ein Teil Rinderserum auf 9 Teile Phosphat-Puffer $0.05 \mathrm{M}, \mathrm{pH} 7.5$ ) gewaschen und jeweils erneut zentrifugiert. Danach wird die Radioaktivität des Präzipitates im Bohrlochkristall gemessen. Die Auswertung erfolgt wie üblich (Konstruktion der Standardkurve, Ablesen der Plasmakonzentration).

\section{Träger-Immunopräzipitat}

Die Aufgabe des Trägerpräzipitates besteht darin, die Menge des während der Zentrifugation sedimentierenden Materials zu vergrößern und evtl. Verluste des Bodensatzes während des Dekantierens relativ zu verringern. Ohne diesen Zusatz ist die Menge an Präzipitat bei der Zusammensetzung der Testkombination des Radiochemical Centre außerordentlich klein. Die Antikörper des vorgegebenen Systems stammen vom Meerschweinchen (Anti-Insulin-Serum) und vom Kaninchen (Antigammaglobulin-Serum). Für das Trägersystem wurde Kaninchen-Gammaglobulin als Antigen gewählt. Antiserum gegen Kaninchengammaglobulin wurde im Schaf erzeugt. Hierzu wurde $4 \mathrm{mal}$ im $\mathrm{Ab}$ stand ron je etwa einer Woche $100 \mathrm{mg}$ Kaninchengammaglobulin (Fraktion II aus Kaninchenplasma,

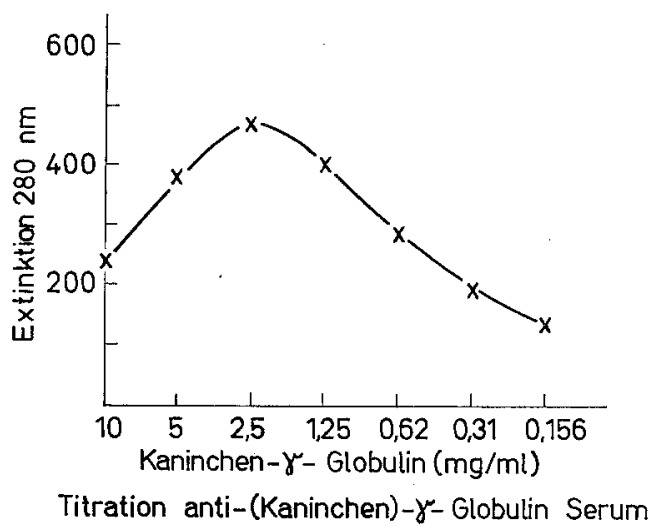

Abb. 3. Titration Anti-(Kaninchen)-Gammaglobulinserum. $0.1 \mathrm{ml}$ Kaninchen-Gammaglobulin der verschiedenen Konzentrationen wurde mit $0.1 \mathrm{ml}$ des Antiserums in einem Gesamt-Volumen von $0.5 \mathrm{ml}$ für $24 \mathrm{Std}$ bei $+4^{\circ} \mathrm{C}$ inkubiert. Weitere Einzelheiten siehe Toxt. Der Äquivalenzpunkt liegt bei $0.1 \mathrm{ml}$ unverdünnten Antiserums/ $0.25 \mathrm{mg}$ Kaninchen-Gammaglobulin

Travenol International GmbH, München) in $0.9 \%$ iger Kochsalzlösung zusammen mit $3-4 \mathrm{ml}$ komplettem Freund'schem Adjuvans subkutan injiziert. Danach war ein ausreichender Antikörpertiter vorhanden, der durch weitere Injektion von je $50 \mathrm{mg}$ Antigen etwa alle 4 Wochen aufrechterhalten wird. Das TrägerAntigen/Antikörpersystem wird vor der Benutzung 
gegeneinander austitriert, um die Konzentrationen der Reaktionspartner so wählen zu können, daB sie im Bereich der Äquivalenzzone liegen und eine optimale Präzipitation erreicht wird (Abb. 3). Für diese Titration wird eine konstante Menge des Antigammaglobulin-Serums $(0.1 \mathrm{ml})$ mit fallenden Konzentrationen des Antigens $(0.1 \mathrm{ml})$ in $0.9 \%$ iger Kochsalzlösung inkabiert (Gesamtvolumen $0.5 \mathrm{ml}, 24$ Std Inkubation bei $+4^{\circ} \mathrm{C}$ ). Anschließend wird zentrifugiert (10 Min. bei etwa $3000 \mathrm{~g}$ ) und zweimal mit je $1 \mathrm{ml}$ Kochsalzlösung nachgewaschen. Das Präzipitat wird in $0.25 \mathrm{~N}$ Essigsäure gelöst und die Eiweißmenge durch Messung der Absorption bei $280 \mathrm{~nm}$ im Spektrophotometer bestimmt. Im Antigen- und im Antikörperüberschuß ist die Präzipitation geringer als in der Aquivalenzzone.

Bei späterer Benutzung von $0.2 \mathrm{ml}$ der Suspension wird eine genügend große Menge an Trägerpräzipitat erzielt, wenn die Lösung des Antigens $40 \mathrm{mg}$ Gammaglobulin $/ 100 \mathrm{ml} 0.9 \%$ iger Kochsalzlösung enthält (um den Faktor 5 bis 10 niedrigere Konzentrationen ergeben ebenfalls zufriedenstellende Präzipitatmengen). Dies entspricht etwa der Gammaglobulinkonzentration eines $1 / 15$ verdünnten Vollserums. Im Beispiel der Abb. 3 liegt die Äquivalenzzone bei $2.5 \mathrm{mg}$ Antigen pro $\mathrm{ml}$ Antiserum. Zur optimalen Präzipitation von $40 \mathrm{mg}$ Antigen wären hier also $16 \mathrm{ml}$ Antiserum zu verwenden (entsprechend $100 \mathrm{ml}$ Antigenlösung der Konzentration $4.0 \mathrm{mg} / 100 \mathrm{ml}$ und $100 \mathrm{ml}$ des $1: 6.25$ verdünnten Antigammaglobulin-Serums).
$0.5 \mathrm{ml}$ bei $-20^{\circ} \mathrm{C}$ aufbewahrt. Je eine Probe wird am Ansatztage aufgetaut und mitbestimmt. Die Differenz der bestimmten Insulinkonzentrationen sollte $50 \mu \mathrm{E} / \mathrm{ml}$ betragen. Die Absolutwerte kontrollieren zugleich die Reproduzierbarkeit von Ansatz zu Ansatz. Zur Kontrolle des Waschrorganges werden Röhrchen angesetzt, die ledighich albuminhaltigen Puffer $(0.1 \%)$ und radioaktiv-markiertes Insulin, jedoch kein Anti-InsulinSerum (Binding Reagent) und kein unmarkiertes Insulin enthalten.

\section{Ergebnisse}

Bei insgesamt 68 verschiedenen Ansätzen wurden mit der beschriebenen Methode folgende Ergebnisse erzielt:

\section{Sensibilität}

Zur Bestimmung der Sensibilität wurde die Signifikanz der Differenz zwischen den Meßwerten berechnet, die zu den Konzentrationen 0 und $10 \mu \mathrm{E}$ Insulin $/ \mathrm{ml}$ bzw. 0 und $20 \mu \mathrm{E}$ Insulin $/ \mathrm{ml}$ der Standardkurven gehören. Werden die Werte aller Standardkurven berücksichtigt ( $n=68$ ), so ist die Differenz der Meßwerte von 0 und $10 \mu \mathrm{E} / \mathrm{ml}$ signifikant $(P<0.05, t$-Verteilung). Wird die Berechnung für Einzelkurven vorgenommen, so ist manehmal erst die Differenz zwischen 0 und $20 \mu \mathbf{E}$ Insulin $/ \mathrm{ml}$ bei der $5 \%$-Grenze signifikant. Es muß also für jeden Ansatz erneut entschieden werden, ob Werte

Tabelle 1. Regressionskoeffizienten (b), Standardabweichung des Regressionskoeffizienten $\left(S_{b}\right)$ und Genauigkeitsindex $(\lambda)$ von fünf einzelnen Standardkurven. Einzelheiten s. Text

\begin{tabular}{lccccc}
\hline & $30.10 .67^{\mathrm{a}}$ & $11.12 .67^{\mathrm{a}}$ & $12.12 .67^{\mathrm{a}}$ & $14.12 .67^{\mathrm{a}}$ & $6.3 .68^{\mathrm{a}}$ \\
\hline$b^{\mathrm{b}}$ & -0.3798 & -0.4326 & -0.5049 & -0.4842 & -0.3868 \\
\hline$S_{b}^{\mathrm{e}}$ & 0.0676 & 0.1359 & 0.0541 & 0.0487 & 0.0258 \\
\hline$\lambda^{\mathrm{d}}$ & 0.1780 & 0.3141 & 0.1071 & 0.1006 & 0.0666 \\
\hline$\lambda^{\mathrm{e}}$ & 0.1974 & 0.2120 & 0.2287 & 0.2206 & 0.1416 \\
\hline
\end{tabular}

a Datumn des Ansatzes

b $b=\frac{S x y}{S_{x}}$

e $S_{b}=\sqrt{\frac{S(y)-b \cdot[S(x y)]}{N-2}}$

d $\lambda=\frac{S_{b}}{b}$ (Ordinate + Abszisse logarithmisch)

e $\lambda=\frac{S_{b}}{b}$ (Ordinate: $\frac{A_{0}}{A_{1}}$ Abszisse logarithmich)

$A_{0}$ und $A_{1}=$ präzipitierte Radioaktivität in Abwesenheit und Gegenwart von unmarkiertem. Insulin

\section{Kontrollen}

Das System wird bei jedem Ansatz in bezug auf Reproduzierbarkeit und Wiederfindung kontrolliert. Hierzu wird ein Poolplasma mit niedrigem Insulingehalt ohne (Plasma I) und mit Zusatz von $50 \mu \mathbf{E}$ Schweine-Insulin/mI (Plasma II) in Portionen von je zwischen 10 und $20 \mu \mathrm{E}$ Insulin/ml wirklich von der Standardkurve ablesbar sind oder nicht.

\section{Genauigkeit}

Die statistische Prüfung der Methode erfolgte weiter durch Berechnung des Genauigkeitsindex $\lambda$ (vgl. 
Legende zu Tab. 1). Entsprechende Berechnungen wurden bisher nur selten angegeben. Um einen Vergleich mit den von Mexani et al. [7] für ihre Methode der radioimmunologischen Insulinbestimmung angegebenen Werte zu ermöglichen, wurden beide Achsen des Koordinatensystems, in das die Werte der Standardkurve eingetragen werden, logarithmisch transformiert (Verhältnis gebundenes zu freiem Insulin (B/F) auf der Ordinate, Konzentration des unmarkierten Insulins auf der Abszisse). Die in Tab. 1 angegebenen Werte zwischen 0.067 und 0.314 sind in 4 von 5 Fällen kleiner als der von Melani et al. angegebene Wert von 0.2 für eine Standardkurve im gleichen Insulinkonzentrationsbereich. Die Schwankungen von $\lambda$ sind weniger stark, wenn die logarithmische Umformung nicht für beide Achsen sondern nur für die Abszisse vorgenommen wird, und die Werte der Ordinate für jede gegebene Insulinkonzentration auf den Wert bei der Insulinkonzentration Null bezogen werden [5]. Dabei wird berücksichtigt, daß bei gegebener Konzentration des Insulin-Antikörpers die maximale Bindung des markierten Insulins in der Abwesenheit unmarkierten Insulins einen endlichen Wert hat (etwa $40-50 \%$ der Gesamtmenge unter den gegebenen Antigen-Antikörper-Verhältnissen der Testkombination des Radiochemical Centre). Der Verlauf der Standardkurve entspricht dann einer Exponential-Funktion und nicht einer Hyperbel. Die nach dieser Umformung errechneten $\lambda$-Werte sind ebenfalls in Tab. 1 aufgeführt.

Bei der Beurteilung der statistischen Berechnungen muß berücksichtigt werden, daß die Sensibilität einer radioimmunologischen Methode u.a. von der Qualität des Antikörpers (seiner Avidität für das Antigen [1]), seiner Konzentration im Ansatz und von der Menge des markierten Antigens abhängig ist. Diese Faktoren sind in der Testkombination des Radiochemical Centre teilweise festgelegt und nicht veränderbar. Es ist also z.B. nicht möglich, durch Verringerung der Konzentration des Anti-Insulinserums und des markierten Insulins die Sensibilität und den Regressionskoeffizienten zu erhöhen und den $\lambda$-Wert zu senken. Der Genauigkeitsindex ist dem Regressionskoeffizienten (Steilheit der Regressionsgraden der Standardkurve) umgekehrt und dessen Standardabweichung direkt proportional (vgl. Legende zu Tab. 1). Da der Regressionskoeffizient im wesentlichen durch die Kombination der Materialen der Testkombination festgelegt ist, könnten durch Vergleiche des Genauigkeitsindex Modifikationen der Methodik in ihrem EinfluB auf die Präzision der Bestimmungen beurteilt werden. Entsprechende Angaben über die Originalmethode, wie sie vom Radiochemical Centre Amersham empfohlen wird, liegen jedoch bisher nicht vor. Die in der Originalarbeit von HaLes and RANDLE [5] angegebenen Werte von $\lambda=0.016$ und 0.056 sind nicht direkt vergleichbar, da offenbar der von ihnen benutzte Antikörper eine steilere Regressionsgerade (stärkerer prozentualer Abfall im Bereich kleiner Konzentrationen unmarkierten Insulins) ermöglichte. Das Gleiche gilt für den von MeLaNI et al. [7] angegebenen Wert von 0.039, der erzielt wurde, als die Standardkurve in den Bereich von 0-25 $\mu \mathrm{E}$ Insulin/ml gebracht werden konnte. Der mit der hier beschriebenen Methode bei einer Standardkurve erzielte Wert von $\lambda=0.0666$ zeigt jedoch, daß diese Genauigkeit mit der Testkombination des Radiochemical Centre unter Benutzung der hier beschriebenen Modifikation prinzipiell auch erreicht werden kann.

\section{Waschkontrollen}

Die Radioaktivität in den Waschkontrollen (Abwesenheit von Anti-Insulin-Serum) beträgt $2.9 \%$ der Gesamtradioaktivität (Standardabweichung $=1.62$ $\mathrm{N}=67)$.

\section{Inkubationszeit}

Die Verlängerung der Inkubationszeiten auf zweimal 24 Std ergab keine Veränderung der absoluten Insulinwerte. Bei mehreren Inkubationen, die mit gleichen Materialien und einerseits den Inkubationszeiten der Originalmethode (6 und 18 Std) andererseits den verlängerten Inkubationszeiten (zweimal $24 \mathrm{Std}$ ) vorgenommen wurden, ergab sich kein signifikanter Unterschied der bestimmten Plasma-Insulinkonzentrationen. Ein solcher Unterschied ist auch prinzipiell nicht zu erwarten, da die Veränderung der Inkubationszeiten Standardkurven und Plasmaproben in gleicher Weise betrifft.

\section{Reproduzierbarkeit und Wiederfindung}

Die Ergebnisse zur Kontrolle der Reproduzierbarkeit und der Wiederfindung sind in Tabelle 2 zusammengestellt. Die Differenz zwischen den Insulinkonzen-

Tabelle 2. Reproduzierbarkeit und Wiederfindung. Einzelheiten s. Text

\begin{tabular}{llll}
\hline & Plasma I & Plasma II & Differenz I/II \\
\hline Insulin $(\mu \mathrm{E} / \mathrm{ml})$ & $25.7^{\mathrm{a}}$ & $78.9^{\mathrm{a}}$ & $53.2^{\mathrm{b}}$ \\
\hline $\mathrm{S}^{\mathrm{c}}$ & 9.9 & 8.1 & 6.1 \\
\hline a Mittelwert aus 10 & Bestimmungen an & verschiedenen \\
Tagen & \\
b Mittelwert der individuellen Differenzen & \\
e S $=\sqrt{\frac{1}{\mathrm{~N}-1}\left[\Sigma\left(x^{2}\right)-\bar{x} \Sigma(x)\right]}$
\end{tabular}

trationen des Plasmas I und des Plasmas II sollte der zugegebenen Menge an Insulin $(50 \mu \mathbf{E} / \mathrm{ml})$ entsprechen. Die relativ große Standardabweichung der Mittelwerte ist zum Teil durch die technischen Gegebenheiten bedingt, auf die in der Besprechung (s. unten) näher eingegangen wird.

\section{Plasma-Insulinkonzentrationen}

Die Plasma-Insulinkonzentrationen von 24 gesunden, normalgewichtigen Erwachsenen im Alter von 
17.5 bis 34 Jahren (13 Frauen und 11 Männer) sind in Tab. 3 angegeben. Aufgeführt sind die Nüchternwerte und zusätzlich die maximale Insulinkonzentration nach Stimulation mit $20 \mathrm{mg}$ Rastinon ${ }^{\circledR} / \mathrm{kg}$ Körpergewicht (i.v.).

Tabelle 3. Nüchternwerte und maximale Insulinkonzentration nach Tolbutamid-( Rastinoni-) Belastung

\begin{tabular}{lllc}
\hline & Nüchtern-1a & Nüchtern-2b & Max.R \\
\hline Insulin $(\mu \mathrm{E} / \mathrm{ml})^{\mathrm{d}}$ & 18.2 & 20.1 & 111.2 \\
\hline$S^{\mathrm{e}}$ & 14.0 & 20.1 & 34.8 \\
\hline $\mathrm{N}$ & 20 & 21 & 14 \\
\hline
\end{tabular}

a 20 Min. vor Rastinongabe

b unmittelbar vor Rastinongabe

c maximale Insulinkonzentration nach Belastung (i.v.) mit $20 \mathrm{mg}$ Rastinon $\otimes / \mathrm{kg}$ Körpergewicht

a Mittelwert $\bar{x}=\frac{\Sigma\left(x_{1}\right)}{N}$

$\mathrm{e} S=\sqrt{\frac{1}{N-1}\left[\Sigma\left(x^{2}\right)-\bar{x} \Sigma(x)\right]}$

\section{Besprechung}

Die Entwicklung der geschilderten Modifikation der Methode von Hares und RandLe wurde von dem Gedanken bestimmt, die Vorteile einer käuflichen Testkombination für die radioimmunologische Insulinbestimmung zu nutzen (Verwendung bereits titrierter Antikörper und radioaktiv-markierten Insulins) und gleichzeitig die Schwierigkeiten bei der B/F-Trennung durch Filtration zu beseitigen. Die Benutzung der Zentrifugation anstelle der Filtration bedeutet eine Arbeitserleichterung (die Zentrifugation ermöglicht die Bearbeitung einer größeren Zahl von Proben und verlangt weniger angestrengte Aufmerksamkeit) und umgeht gleichzeitig alle Schwierigkeiten, die sich durch ungleiche Filterqualität [6] oder durch Adsorption an die Filter ergeben können. Die geringe Menge an Präzipitat, die bei der Zusammensetzung der Testlkombination des Radiochemical Centre erzielt wird, mußte jedoch für die Zwecke der Zentrifugation vergrößert werden, am evtl. Verluste beim Waschen relativ zu verringern. Das hierzu benutzte präzipitierende Antigen/Antikörpersystem (Trägerpräzipitat) kann wahrscheinlich auch von anderen als den benutzten Spezies stammen und muß nicht unbedingt Gammaglobulin als Antigen benutzen. Fs muß jedoch eine Interferenz mit dem eigentlichen Bestimmungssystom vermieden werden. So müssen auch alle Stoffe, die das Präzipitat in unspezifischer Weise vergrößern könnten (Latex, Zellulose oder ähnliches) aber evtl. freies Insulin adsorbieren, vermieden werden.

Die mit dem modifizierten System erzielten Ergebnisse entsprechen etwa denen einiger anderer Methoden zur radioimmunologisehen Insulinbestimmung. Die Sensibilität der Standardkurve erscheint ausreichend, wenn sie $10 \mu \mathrm{E}$ Insulin/ml erreicht, da die normalen
Nüchternwerte der Insulinkonzentration im menschlichen Plasma im allgemeinen bei etwa $20 \mu \mathrm{E} / \mathrm{ml}$ liegen (s. unten). Bei der hier angegebenen Sensibilität ist zu berücksichtigen, daß aus technischen Gründen die Messung der Radioaktivität im Bohrlochkristall and mit zwei ineinandergestellten Glasröhrchen vorgenomwurde. Bei der relativ weichen $\gamma$-Strahlung des $J^{12 s}$ (35 $\mathrm{keV}$ ) entstehen hierbei erhebliche StrahlungsverIuste durch unterschiedliche Glasstärken und in der Luft. Es ist anzunehmen, daB die Signifikanz der MeBwertdifferenzen wesentlich verbessert werden kann, wenn entweder im Flüssigkeits-Szintillationszähler gemessen wird oder wenn $J^{131}$ anstelle von $J^{125}$ benutzt wird4. Literaturangaben über die Sensibilität anderer radioimmunologischer Insulinbestimmungsmethoden sind etwa $20 \mu \mathrm{E} / \mathrm{ml}[4], 6.5 \mu \mathrm{E} / \mathrm{ml}$ [2], $0.1 \mu \mathrm{E} / \mathrm{ml}[11]$.

Die Reproduzierbarkeit entspricht etwa der von Buchanan und MoKIonte [2] ebenfalls mit der Methode von HaImS und RaNDLit erzielten. Die Wiederfindung ist mit 106\% der eingesetzten Insulinmenge $(53.2 \mu \mathrm{E} / \mathrm{ml}$ anstelle von $50 \mu \mathrm{E} / \mathrm{ml})$ zufriedenstellend. Die gefundenen Insulin-Plasmakonzentrationen entsprechen den von anderen Autoren bei vergleichbaren Kollektiven gefundenen Werten. Angaben für InsulinNüchternwerte sind z.B. $21 \mu \mathrm{E} / \mathrm{ml}[11], 17.2 \mu \mathrm{E} / \mathrm{ml}[5]$, $22 \mu \mathrm{E} / \mathrm{ml}(10)$ and $20 \mu \mathrm{E} / \mathrm{ml}$ [3]. Der Anstieg nach Rastinon-Injektion ist allerdings größer als der von anderen Autoren beschriebene [8, 9], jedoch sind die Werte untereinander nicht vergleichbar, da die verwendete Rastinon-Dosis verschieden groß ist $(1 \mathrm{~g} \mathrm{bzw}$. $20 \mathrm{mg} / \mathrm{kg}$ Körpergewicht).

Toh danke Herrn Prof. Dr. G. Fucrs (Direktor des Institutes für Med. Statistilk und Dokumentation) für wertvolle Hilfe bei den statistischen Berechnungen.

Die Farbwerke Hoechst AG, Frankfurt/Main, stollten freundlicherweise kristallines Rinder- und SchweineInsulin zur Verfügung.

Die Arbeiten wurden mit technischer Hilfe von Fräulein INGe Qurrzow und Früulein KanTN DoratKowskI durchgeführt.

Die Untersuchungen wurden durch die Deutsche Forschungsgemeinschaft gefördert.

\section{Literatur}

1. Berson, S.A., and R.S. Yaxow: In: The Hormones, Vol. IV, S. 557 ff. (G. Privers, K.V. ThImann, E.B. Astwooo Edts.) Now York/London: Academic Press, 1964.

2. BuchanaN, K.D., and M.T. McKmDIE: Experience with the Immunoprecipitation Technique of Insulin Assay with Reference to Sensitivity, Precision and Specificity. Clin. chim. Acta 15, 315-320 (1967).

4 Seit der Fertigstellung dieser Arbeit wurde ein anderes Meßgerät (Baird-Atomic, Isomatic) benutzt, in dem Plastikröhrehen zur Anwendung kommen. Die engere Bohrung des Kristalls verringert außerdem die Stärke der Luftschicht. Die jetzt erzielten Meßwerte weisen eine erheblich geringere Streubreite auf. 
3. - The Normal Tnsulin Response to Glucose. Diabetologia 3, 460-464 (1967).

4. Grodsky, G.M., and P.H. Forsham: An Immunochemical Assay of Total Extractable Insulin in Man. J. clin. Invest. 39, 1070-1079 (1960).

5. HATES, C.N., and P.J. RANDL: Immunoassay of Insulin with Insulin-Antibody Precipitate. Biochem. J. 88, 137-146 (1963).

6. Hartog, M., M.A. GaAfar, B. Meisser, and R. FraSER: Immunoassay of Serum Growth Hormone in Acromegalic Patients. Brit. med. J. 1964 II, 12291232.

7. Merlant, F., H. Dirschuneit, K.M. Barteut, H. Frimorich u. H.F. PreIffer: Ûber die radioimmunologische Bestimmung von Insulin im Blut. Klin. Wschr. 43, 1000-1007 (1965),

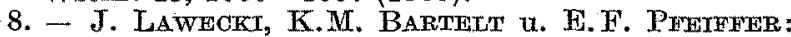
Immunologisch meBbares Trosulin (IMI) bei Stoffwechselgesunden, Fettsüchtigen und adipösen Diabetikern nach intravenöser Gabe von Glucose, Tolbutamid und Glucagon. Diabetologia 3, 422-426 (1967).

9. Pertery, M., and D.M. KrpNrs: Effect of Glucocorticoids on Plasma-Insulin. New Engl. J. Med. 274, $1237-1241(1966)$.

10. Rundtischgespräch über die radioimmunologische Bestimmung von Insulin. 14. Symposion der Deutschen Gesellschaft für Endokrinologio (Heidelberg 1968), im Druck.

11. YAIOW, R.S., and S.A. BersoN: Immunoassay of Findogenous Plasma Insulin in Man. J. clin. Invest. 39 . $1157-1175(1960)$.

Dr. H.-J. QUABBe

II. Medizinische Universitätsklinik der Freien Universität Berlin 1000 Berlin 19

Spandauer Damm 130 